\title{
A Public Health Informatics Solution to Improving Food Safety in Restaurants: Putting the Missing Piece in the Puzzle
}

\author{
Melanie J. Firestone ${ }^{1 *}$, Sripriya Rajamani ${ }^{2}$, Craig W. Hedberg ${ }^{1}$ \\ ${ }^{1}$ University of Minnesota, School of Public Health, Division of Environmental Health Sciences, \\ Minneapolis, MN, USA \\ ${ }^{2}$ University of Minnesota, Institute for Health Informatics and School of Nursing, Minneapolis, MN, USA; \\ Project Consultant, Minnesota Department of Health, St. Paul, MN, USA
}

\begin{abstract}
Foodborne illnesses remain an important public health challenge in the United States causing an estimated 48 million illnesses, 128,000 hospitalizations, and 3,000 deaths per year. Restaurants are frequent settings for foodborne illness transmission. Public health surveillance - the continual, systematic collection, analysis, and interpretation of reports of health data to prevent and control illness - is a prerequisite for an effective food control system. While restaurant inspection data are routinely collected, these data are not regularly aggregated like traditional surveillance data. However, there is evidence that these data are a valuable tool for understanding foodborne illness outbreaks and threats to food safety. This article discusses the challenges and opportunities for incorporating routine restaurant inspection data as a surveillance tool for monitoring and improving foodborne illness prevention activities. The three main challenges are: 1) lack of a national framework; 2 ) lack of data standards and interoperability; and 3) limited access to restaurant inspection data. Tapping into the power of public health informatics represents an opportunity to address these challenges. Advancing the food safety system by improving restaurant inspection information systems and making restaurant inspection data available to support decision-making represents an opportunity to practice smarter food safety.

Keywords: restaurant inspections, public health informatics, surveillance data, environmental health, information systems

*Correspondence: Melanie J. Firestone, fire0018@umn.edu

DOI: 10.5210/ojphi.v13i1.11087

Copyright (C2021 the author(s)

This is an Open Access article. Authors own copyright of their articles appearing in the Online Journal of Public Health Informatics. Readers may copy articles without permission of the copyright owner(s), as long as the author and OJPHI are acknowledged in the copy and the copy is used for educational, not-for-profit purposes.
\end{abstract}




\section{BACKGROUND}

Foodborne illnesses remain an important public health challenge in the United States. Each year, an estimated 48 million people get sick, 128,000 are hospitalized, and 3,000 die from foodborne illness in the United States [1,2] resulting in an annual estimated cost of \$51 billion [3]. Reducing the occurrence of foodborne illness infections is a Healthy People 2030 objective [4]. Achieving this objective requires targeted strategies across the food system. Restaurants are linked to outbreaks more often than other places of food preparation, accounting for two-thirds of outbreaks in 2017 [5]. More than 3,000 state, local, and tribal agencies are responsible for regulating the more than 1 million food establishments in the United States [6]. Routine public health inspections of restaurants are conducted to identify and correct food handling errors within individual restaurants.

Public health surveillance - the ongoing, systematic collection, analysis, and interpretation of reports of health data to prevent and control disease and illness - is a prerequisite for an effective food control system [7]. Traditionally, restaurant inspection data have not been aggregated for use as a hazard surveillance tool to identify food safety gaps and to inform foodborne illness prevention activities because these data are often not stored in ways that allow for real-time analysis. In addition, inspection conditions at a particular point of time that are either corrected immediately or shortly after the inspection are not collected. This treatment of violations as discrete events rather than as indicators of underlying trends leads to a lack of understanding in how these data relate to foodborne illness risk [8]. However, access to data on restaurant inspections could provide added value to existing food surveillance schemes and enhance food safety. The current COVID19 pandemic highlights the need for better management of public health data that links the occurrence of illness to resources needed to control transmission [9]. As the United States recognizes and works toward improving public health data systems, there is an opportunity to include advancements that support enhanced food safety prevention activities.

Public health informatics (PHI) is defined as the systematic application of information, computer science and technology to public health practice, research and learning [10]. A recent update defines PHI by the effective use of information and information technology to improve population health outcomes [11]. The practice of public health is increasingly recognized as being data and information intensive [12,13]. There is a need for "informatics-savvy" health departments [14,15] where informatics is a strategic priority [16]. In recent decades, the use of electronic health records (EHRs) has increased. Although the primary goal of EHRs is to improve clinical practice, EHRs provide a low-cost and timely means of accessing rich data sources for population health surveillance [17]. Investment in EHRs, syndromic surveillance, and electronic case reporting (eCR), and electronic laboratory reporting (ELR) have greatly advanced population health surveillance for both chronic and infectious diseases [18]. In a similar manner, creating a framework to connect restaurant inspection data to surveillance for individual cases and outbreaks of foodborne illness could directly enhance public health efforts to reduce transmission of illness in restaurant settings.

The framework for an evolved public health system, referred to as Public Health 3.0 emphasizes leveraging cross-sector collaboration and environmental, policy, and system-level approaches to directly impact social determinants of health [19,20]. While Public Health 3.0 includes a focus on 
timely and locally relevant health information systems, challenges remain in terms of people, policies and politics to realize the full potential. In this paper, we present challenges to incorporating restaurant inspection data in the overall schema of food safety and surveillance: 1) lack of a national framework to integrate restaurant inspection data into other foodborne illness surveillance systems, 2) lack of data standards and interoperability between information systems operated by public health and food regulatory agencies at local, state and federal levels, and 3) limited access to restaurant inspection data by regulators, public health researchers and consumers. These hurdles can be addressed by tapping into the power of PHI. We advocate for advancing the field of PHI to bring in missing pieces of data to connect the dots for betterment of food surveillance and safety. Lessons learned from COVID-19 are applicable to foodborne illness surveillance. As upgrades to the public health infrastructure are considered, food safety systems and surveillance should be part of those considerations.

\section{LANDSCAPE: RESTAURANT INSPECTION DATA AND CHALLENGES}

The Food and Drug Administration (FDA) publishes the Food Code, an evidence-based, voluntarily-adopted model that provides jurisdictions with a technical and legal basis for regulating retail food service [21]. While the Food Code provides uniform national standards for retail food safety, it has not been adopted nationally. Local, state, and tribal regulators use this as a model to develop or adopt their own food safety regulations. Since 2014, more than half of money spent on food each year in the US is spent on food prepared away from home [22]. Given that food prepared away from home is an integral part of the diet in the US, there is a need to monitor food safety risks associated with it.

Under current COVID-19 pandemic circumstances, restaurants have been a key social setting to be monitored. Restaurants were ordered to comply with various directives imposed by their local jurisdictions and states to reduce transmission of SARS-CoV-2, the virus that causes COVID-19. Restaurants have gone through various phases of adapting to this new landscape. While some restaurants have closed completely others have shifted to operating on a take-out or delivery only model and some have opened with adherence to socially distanced seating, mask mandates and relevant public health guidance, or a combination of these strategies depending on local COVID19 prevention measures. As public health measures are adapted to local transmission patterns of COVID-19, there may be critical challenges associated with restoring supply chains and inspection practices. Most inspections serve primarily an operational or administrative function. Recently inspectors have had an increased role in enforcement in many areas for compliance with COVID19 prevention measures. The diverse array of inspection data systems in use do not support the needs of public health practice research. The lack of interoperability between these restaurant inspection data systems means that we do not have the ability to assess critical threats to restaurant food safety in this unprecedented time.

\section{A. Lack of a National Framework}

Surveillance for foodborne illnesses is a complex, multi-faceted endeavor coordinated by multiple agencies across federal, state and local levels in the United States. Individual cases of reportable foodborne illness and suspected outbreaks of foodborne illness are reported to local and state health departments under state-specific reportable disease rules. Many foodborne illnesses, 
including, Salmonella and Shiga toxin-producing Escherichia coli (STEC) infections are nationally notifiable conditions that are monitored through the National Notifiable Diseases Surveillance System (NNDSS) at the Centers for Disease Control and Prevention (CDC) [23]. Foodborne illness outbreaks are reported to the National Outbreak Reporting System (NORS).

While authority for disease investigation resides with state and local agencies, CDC plays a critical coordinating and capacity building role. CDC's principal method for helping to build this public health capacity is the Epidemiological and Laboratory Capacity (ELC) Cooperative Agreement program, which provides funding to 64 participating jurisdictions, including all 50 states, three freely associated states, five territories, and six local governments.

This funding supports the staff, supplies, training, and equipment needed for public health departments to participate in nationwide surveillance networks. PulseNet, a national network of public health and food regulatory laboratories that provides molecular characterization of important foodborne pathogens demonstrates the value of a national framework for foodborne illness prevention. By working together to rapidly detect foodborne illness outbreaks across the country, Pulsenet has led to the prevention of an estimated 270,000 illnesses every year from Salmonella, E. coli $\mathrm{O} 157$ and Listeria monocytogenes. This was estimated to save $\$ 507$ million in medical costs and lost productivity, an economic benefit at least 70 times greater than the cost [24].

Although restaurants are a frequent setting for foodborne illnesses and outbreaks, there is no coordinated surveillance system for food safety hazards identified by routine restaurant inspections, like PulseNet. The Centers for Disease Control and Prevention maintains the National Environmental Assessment Reporting System (NEARS), which captures environmental assessment data from foodborne illness outbreak investigations [25]. Environmental assessments differ from routine inspections because they are targeted inspections that seek to identify how and why a foodborne illness outbreak occurred with the goal of identifying opportunities for prevention. Review of routine inspection reports may provide additional useful information for identifying the causes of outbreaks and prevention opportunities.

The FDA Retail Food Risk Factor Study is a 10-year study to measure the occurrence of foodborne illness risk factors, food safety practices and behaviors, and interventions in food service facilities. The goal of this study is to provide consistent monitoring of food safety trends and efforts over time. Trained data collectors observe and record food safety practices in restaurants using a standardized tool. However, the survey is episodic, only a subset of restaurants in the US are studied and the generalizability is limited to the extent that facilities in the sampling zones are generalizable to the overall industry [26].

A NEARS review of 9,788 restaurant-associated outbreaks showed that most commonly reported contributing factors were associated with food handling and preparation practices [27]. The Center for Science in the Public Interest examined more than 500 restaurant inspection reports in 20 cities and found that over $66 \%$ of restaurants had at least one high-risk violation [28]. Data from 821 restaurants in the 2013-14 FDA Retail Food Risk Factor Study, showed that $86 \%$ of restaurants had violations for improper food holding/time and temperature, and $75 \%$ had violations for poor personal hygiene, both of which are major risk factors for foodborne illness transmission in 
restaurants [26]. While these data show that major risk factors for foodborne illness transmission are commonly cited, there is no national standard for tracking, monitoring, or maintaining these vital data that could be used to monitor food safety risks more broadly or to evaluate the effectiveness of food safety interventions.

\section{B. Lack of Data Standards and Interoperability}

\section{B.1. Lack of Data Standards and Interoperability}

Apart from the issue of lack of a robust, national restaurant inspection data information system, another hurdle is a lack of standardization of both inspection practices, the inspection reports, and the relevant data. Most health departments divide inspection violations into two or three categories based on the risk that the violation could lead to foodborne illness transmission. Currently, the Food Code categorizes violations into three types based on importance - priority, priority foundation, and core [29]. Prior to the 2009 Food Code, violations were categorized as either being "critical" or "non-critical" [30]. As of the end of 2018, 10 states had at least one state regulatory agency that was using a version of the Food Code older than 2009 [31]. Jurisdictions may also choose to use other language to categorize violations. For example, Minnesota uses 3 categories Priority 1, Priority 2, or Priority 3 [32]. This shows that even among states that have adopted the voluntary Food Code, there is considerable variation in language used across jurisdictions.

Another issue to address is the lag in development and adoption of standards related to data in an inspection report. The FDA recognized the lack of national uniformity among retail food safety programs and started the Voluntary National Retail Food Regulatory Program Standards initiative with the goal of defining what constitutes highly effective and responsive retail food programs. As of January 2020, there were 865 enrollees, however, there is considerable variability in levels of conformance with the nine program standards [33]. Since this program is voluntary, there are still broad practical challenges for analyzing inspection data across programs. We recently conducted a study that evaluated restaurant inspection data in the context of an outbreak [8] in Minnesota. This study affirmed the issue of lack of standardization in inspection reports. Violations were abstracted from inspection reports from 13 jurisdictions and were mapped to the Conference for Food Protection's inspection report structure due to the variation in the inspection forms used across jurisdictions, even though all jurisdictions were within the same state. Overall, there is a lack of standards for restaurant inspections that limits the ability to compare violations across jurisdictions. In addition, there is a lack of national standards to support the exchange of data across systems.

\section{B.2. Lack of Scalable Information Systems}

Inspection data are rarely organized like other surveillance systems. While NEARS maintains national data from environmental assessments, there is no national surveillance structure for routine restaurant inspections. There is considerable variation in how routine inspection data are maintained across jurisdictions. Some maintain analyzable databases, others have scanned copies of PDFs, and some maintain only paper records. Among jurisdictions that maintain analyzable databases, a lack of interoperability across these systems limits the ability to monitor food safety hazards across jurisdictions. Jurisdictions without analyzable restaurant inspection databases lack 
the ability to compare and monitor food safety hazards both within their jurisdictions and across other jurisdictions.

\section{Limited Access to Restaurant Inspection Data}

\section{C.1. Access for Consumers}

Restaurant inspection reports are public data, but there is considerable variation in public disclosure practices. Some jurisdictions choose to not actively release restaurant inspection results to the public, while others share them online, in the news, or at restaurants themselves. In recent years, numerous jurisdictions have adopted or considered publicly posting results. Los Angeles County began to publicly post inspection results in restaurant windows so that they are visible prior to entry in 1998 and New York City followed suit in 2010. However, the format of disclosure can vary greatly even among jurisdictions that publicly post results. For example, North Carolina publicly posts a numerical score in addition to letter grades. Rather than being posted so that they are visible before entry, they are posted within restaurants so that they are visible upon entry. Furthermore, an A grade in one jurisdiction may not have the same meaning as an A grade in another jurisdiction, which poses a challenge for consumer understanding.

\section{C.2. Access for Regulators/Public Health Researchers}

Restaurant inspection results can provide valuable information to understand the root causes of foodborne illness, to monitor food safety hazards and to evaluate the effectiveness of inspection programs. In New York City, inspection data are collected on handheld computers using standardized forms and the data is transmitted nightly. Analysis of these data suggested that existing program incentives were not leading to improvements in sanitary conditions. The existence of a data system that enabled data analysis allowed for the detection of a problem and was used to inform the design of a new inspection system (letter grading) and later the evaluation of the new system [35].

Furthermore, our research suggests that valuable information can be gleaned from routine inspection reports as surveillance data to help understand why outbreaks occur [8]. If analyzed during an outbreak investigation, routine restaurant inspection data can supplement traditional surveillance data, which could be useful for identifying why and how an outbreak occurred in addition to what caused the outbreak.

Access to data for research and regulatory purposes can yield valuable insights and we see this action with the ongoing COVID-19 pandemic. Case data is being linked with travel history, exposures, pre-existing conditions, health insurance and other socio-economic indicators to present various perspectives on risk factors and outcomes. Research enables the use of novel methods like data mining to discover patterns in the data, machine learning to predict, and applications of models to simulate transmission and project public health impact and disease spread. 


\section{ADVANCING THE FOOD SAFETY SYSTEM}

\section{Support Robust Public Health Data Infrastructure}

A lack of coordination, a general lack of integrated data, and the inability to analyze data rapidly were recognized as some of the major challenges for a coordinated U.S. response to the COVID19 pandemic [9]. Prior to the pandemic, funding was requested to support the CDC's Data Modernization Initiative which aims to update the agency's core data reporting, analysis and surveillance capabilities to better track emerging health threats. A recent report by the Council of the State and Territorial Epidemiologists (CSTE) advocated for a "public health data superhighway" that is based on a robust core public health data infrastructure that supports automatic and interoperable data exchange [36]. The success of PulseNet in preventing foodborne illnesses demonstrates the value of a coordinated national framework for disease prevention. There is an opportunity to include restaurant inspection data to the foodborne illness surveillance that adds the missing piece of the puzzle (Figure 1).
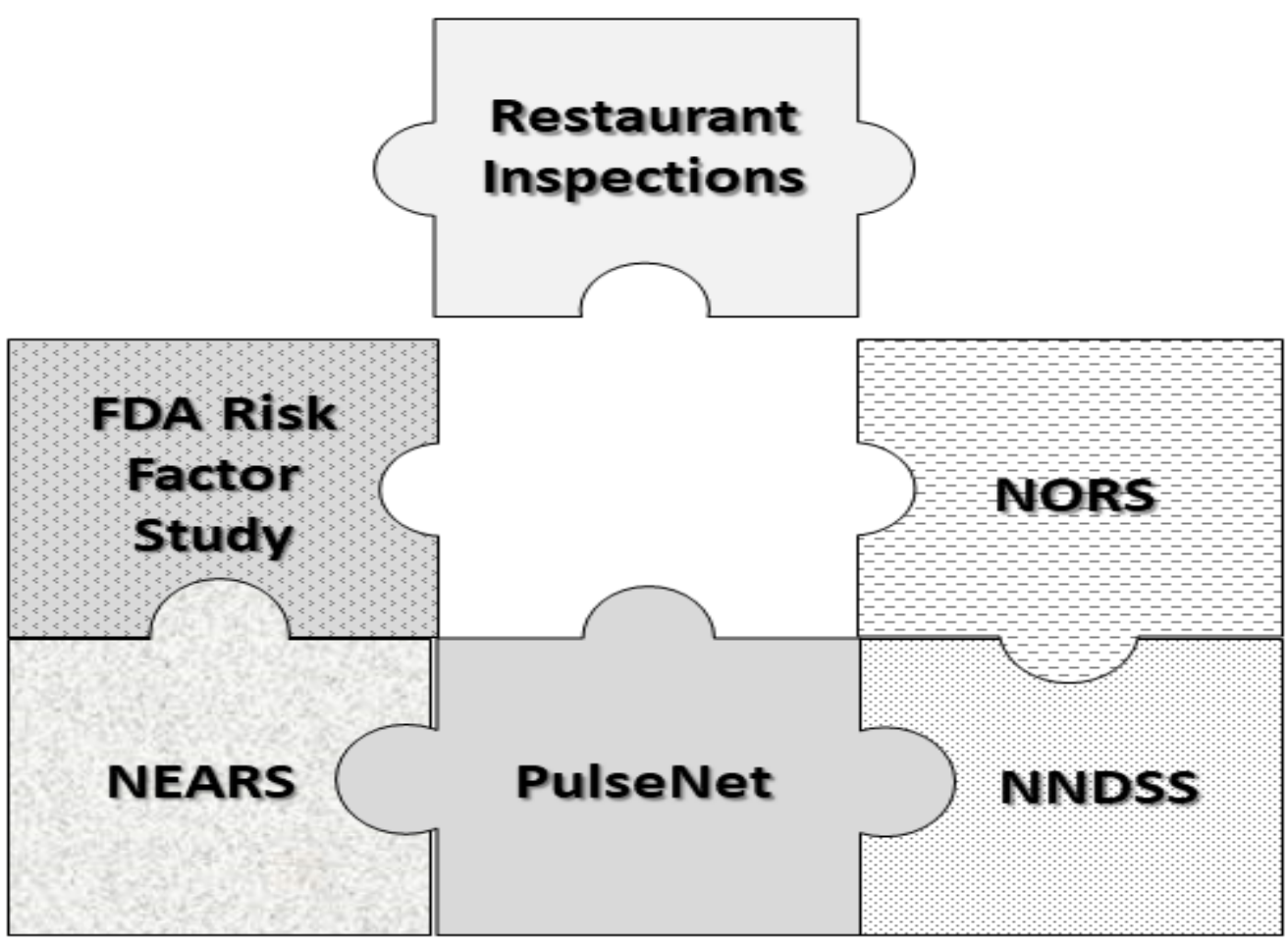

Figure 1: Food-borne Illness Surveillance Systems and the Missing Piece in the Puzzle

There is a need to examine the role of informatics across the spectrum - information and communication technologies can support public health surveillance in a multitude of ways ranging from prevention, detection, and response, laboratory reporting, push notification, analytics and predictive surveillance [37]. The overwhelming COVID-19 news coverage highlights the need for public health officials to have reliable tools built on an interoperable information infrastructure. This pandemic must be a "wake-up call" for investing in the needed resources [38]. An unprecedented opportunity is presented to public health through passage of CARES Act [39] and 
much needed funding to embark on this informatics journey. These investments can have spillover effects on food safety and there is an opportunity to advance the food safety surveillance systems along with other public health information systems. An overarching strong data and information systems backbone will help food safety surveillance by making it feasible for restaurant inspection data to be available in timely fashion.

\section{Promote Standards and Interoperability}

Imagine data coming in electronically (not over phone/fax/paper) not just for COVID-19, but also for foodborne illness cases and outbreaks. This is feasible only with the standards for transmission of data which support transactions such as electronic case reporting [36]. Now let us take it further by integrating restaurant inspection data into disease surveillance systems in real-time to support epidemiological decision making. These efficient and interoperable data flows are not just fiction but made feasible through informatics. The adoption of standards for representation of data will ensure the "apples-to-apples" comparison across jurisdictions. There is currently a lack of standards across the board: lack of uniformity in data collection, lack of standardized data representation and a lack of standards in transmission of data across systems. While the Voluntary National Retail Regulatory Program Standards is an important step for promoting standards, there is still a need for a widespread adoption, interoperability of data systems and a national framework for tracking and monitoring hazards in restaurants.

\section{Advocate Open Data Movement}

There is an increasing trend to make the government data accessible for public utility. Many agencies from the U.S. Department of Health and Human Services (Centers for Medicare and Medicaid Services, CDC, FDA, Agency for Health Care Research and Quality) and other state partners are making data available at Healthdata.gov [40]. Of the 4,280 datasets posted as of April 1,2020 , only 10 pertain to restaurant inspection data. These datasets represent only 7 jurisdictions ( 2 states, 1 county, 1 multi-county, and 3 cities). Some jurisdictions do also provide restaurant inspection data on their own respective websites with the goal of increasing transparency. However, this practice is not a national standard and there is a need to advocate for making data available more broadly.

\section{Empower Practitioners, Researchers and Consumers}

COVID-19 has also highlighted the power of data and making it accessible for variety of interested parties in a timely manner. Integration of data related to foodborne illness surveillance - data from traditional disease reporting methods bolstered by restaurant inspection data along with consumer data - social media reviews of restaurants [e.g. Yelp [41,42], feeds on a particular restaurant [e.g. Twitter $[43,44]$ and novel data [45] immensely enable the ability of practitioners to understand the many facets of an outbreak. Researchers can utilize this to determine causalities, role of risk factors and varying outcome trajectories across population. Consumers are empowered as their data is used for decision-making and in turn can influence the behaviors of other consumers and the restaurants. 


\section{CONCLUSIONS}

Public Health 3.0 emphasizes cross-sector collaboration and environmental, policy and systemslevel actions and underscores the role of technology, tools and data [19,20]. This holistic view should serve as impetus for better food safety surveillance. A broad emphasis on informatics is needed to empower public health decisions through state-of-the-art information systems, high quality data and better workforce capacity. In April 2019, the FDA announced steps to usher in a new era of smarter food safety that leverages technology and other tools to create a more digital, traceable food safety system. CDC's partnership with other federal agencies, Health Information Technology (HIT) vendors and associations is essential to cover the scope of food safety and surveillance. The COVID-19 pandemic underscores the need for a framework of data, information, knowledge, wisdom and practice, and it is more important than ever to appreciate the power of data. Now is the time to push for an overarching food safety surveillance framework which incorporates restaurant inspection data as an essential ingredient and put this missing piece in the puzzle.

\section{FINANCIAL DISCLOSURE}

The authors do not report any financial disclosures and declare that the research was conducted in the absence of any commercial or financial relationships.

\section{COMPETING INTERESTS}

The authors do not have any competing interests.

\section{REFERENCES}

1. Scallan E, et al. 2011. Foodborne Illness Acquired in the United States-Unspecified Agents. Emerg Infect Dis. 17(1), 16-22. PubMed https://doi.org/10.3201/eid1701.P21101

2. Scallan E, et al. 2011. Foodborne illness acquired in the United States--major pathogens. Emerg Infect Dis. 17(1), 7-15. PubMed https://doi.org/10.3201/eid1701.P11101

3. Scharff RL. 2012. Economic burden from health losses due to foodborne illness in the United States. J Food Prot. 75(1), 123-31. PubMed https://doi.org/10.4315/0362-028X.JFP$\underline{11-058}$

4. Healthy People 2030. Food Safety. 2021 [cited 2021 January 10]; Available from: https://www.healthypeople.gov/2020/topics-objectives/topic/food-safety/objectives.

5. Centers for Disease Control and Prevention. Surveillance for Foodborne Disease Outbreaks, United States, 2017, Annual Report. 2019, U.S. Department of Health and Human Services, CDC: Atlanta, GA. 
6. Food and Drug Administration. Retail Food Protection. 2020 [cited 2020 March 29]; Available from: https://www.fda.gov/food/guidance-regulation-food-and-dietarysupplements/retail-food-protection.

7. Tauxe RV. 2002. Surveillance and investigation of foodborne diseases: roles for public health in meeting objectives for food safety. Food Control. 13, 363-69.

https://doi.org/10.1016/S0956-7135(01)00091-3

8. Firestone M, et al. 2020. Can aggregated restaurant inspection data help us understand why individual foodborne illness outbreaks occur? J Food Prot. 83 (5) PubMed https://doi.org/10.4315/JFP-19-576

9. Dixon BE, Caine VA, Halverson PK. 2020. Deficient Response to COVID-19 Makes the Case for Evolving the Public Health System. Am J Prev Med. 59(6), 887-91. PubMed https://doi.org/10.1016/j.amepre.2020.07.024

10. Yasnoff WA, et al. 2000. Public Health Informatics: Improving and Transforming Public Health in the Information Age. J Public Health Manag Pract. 6(6), 67-75. PubMed https://doi.org/10.1097/00124784-200006060-00010

11. Public Health Informatics Institute (PHII). Defining Public Health Informatics. 2020 [cited 2020 March 29]; Available from: https://phii.org/defining-public-health-informatics.

12. Baker EL, et al. 2016. Building the Business Case for Public Health Information Systems. $J$ Public Health Manag Pract. 22(6), 603-06. PubMed https://doi.org/10.1097/PHH.0000000000000495

13. Baker EL, et al. 2016. What Is "Informatics"? J Public Health Manag Pract. 22(4), 420-23. PubMed https://doi.org/10.1097/PHH.0000000000000415

14. LaVenture M, et al. 2014. Building an informatics-savvy health department: part I, vision and core strategies. J Public Health Manag Pract. 20(6), 667-69. PubMed https://doi.org/10.1097/PHH.0000000000000149

15. LaVenture M, et al. 2015. Building an informatics-savvy health department II: operations and tactics. J Public Health Manag Pract. 21(1), 96-99. PubMed https://doi.org/10.1097/PHH.0000000000000179

16. Lovelace, K. and G.H. Shah, Informatics as a Strategic Priority and Collaborative Processes to Build a Smarter, Forward-Looking Health Department. J Public Health Manag Pract, 2016. 22 Suppl 6, Public Health Informatics: p. S83-S88.

17. Casey JA, et al. 2016. Using Electronic Health Records for Population Health Research: A Review of Methods and Applications. Annu Rev Public Health. 37, 61-81. PubMed https://doi.org/10.1146/annurev-publhealth-032315-021353 
18. Association of State and Territorial Health Officials. Leading Public Health Practice Through Health Informatics and Technology. 2018 [cited 2020 January 3]; Available from: https://www.astho.org/ASTHOReports/ASTHO-Informatics-Guidebook/12-14-18/.

19. DeSalvo, K. and Y.C. Wang, Health Informatics in the Public Health 3.0 Era: Intelligence for the Chief Health Strategists. J Public Health Manag Pract, 2016. 22 Suppl 6, Public Health Informatics: p. S1-S2.

20. DeSalvo KB, et al. 2016. Public Health 3.0: Time for an Upgrade. Am J Public Health. 106(4), 621-22. PubMed https://doi.org/10.2105/AJPH.2016.303063

21. Food and Drug Administration. FDA Food Code. 2019 [cited 2021 January 3]; Available from: https://www.fda.gov/food/retail-food-protection/fda-food-code.

22. Saksena MJ, et al. America's Eating Habits: Food Away from Home. 2018, U.S. Department of Agriculture, Economic Research Service.

23. Centers for Disease Control and Prevention. 2020 National Notifiable Conditions. [cited 2020 April 7]; Available from: https://wwwn.cdc.gov/nndss/conditions/notifiable/2020/.

24. Scharff RL, et al. 2016. An Economic Evaluation of PulseNet: A Network for Foodborne Disease Surveillance. Am J Prev Med. 50(5) (Suppl 1), S66-73. PubMed https://doi.org/10.1016/j.amepre.2015.09.018

25. Centers for Disease Control and Prevention. National Environmental Assessment Reporting System (NEARS). [cited 2020 April 7]; Available from:

https://www.cdc.gov/nceh/ehs/nears/index.htm.

26. Food and Drug Administration. FDA Report on the Occurrence of Foodborne Illness Risk Factors in Fast Food and Full-Service Restaurants, 2013-2014. 2018 [cited 2020 February 14]; Available from: https://www.fda.gov/media/117509/download.

27. Lipcsei LE, et al. 2019. Foodborne illness outbreaks at retail establishments - National Environmental Assessment Reporting System, 16 state and local health departments, 20142016. MMWR Surveill Summ. 68(No. SS-1), 1-20. PubMed https://doi.org/10.15585/mmwr.ss6801a1

28. Center for Science in the Public Interest (CSPI). Dirty Dining: Have Reservations? You Will Now. 2008 [cited 2020 April 2]; Available from: www.cspinet.org/new/pdf/ddreport.pdf.

29. Food and Drug Administration. Food Code. 2017 [cited 2020 April 30]; Available from: https://www.fda.gov/media/110822/download.

30. Food and Drug Administration. Food Code. 2009 [cited 2020 April 30]; Available from: https://wayback.archive-

it.org/7993/20170406184540/https:/www.fda.gov/downloads/Food/GuidanceRegulation/U CM189448.pdf. 
31. Food and Drug Administration, Adoption of the FDA Food Code by State and Territorial Agencies Responsible for the Oversight of Restaurants and Retail Food Stores. 2018.

32. Minnesota Department of Health. 20 Questions: The Major Changes of Concern to the Minnesota Food Code. [cited 2020 April 12]; Available from:

https://www.health.state.mn.us/communities/environment/food/docs/20qstnchge.pdf.

33. Food and Drug Administration. Listing of Jurisdictions Enrolled in the Voluntary National Retail Food Regulatory Program Standards. 2020 [cited 2020 April 13]; Available from: https://www.fda.gov/food/voluntary-national-retail-food-regulatory-programstandards/listing-jurisdictions-enrolled-voluntary-national-retail-food-regulatory-programstandards.

34. Food and Drug Administration. Voluntary National Retail Food Regulatory Program Standards - January 2019. 2019 [cited 2020 April 13]; Available from: https://www.fda.gov/media/86636/download.

35. Jeffery NL, McKelvey W, Matte T. 2015. Using tracking infrastructure to support public health programs, policies, and emergency response in New York City. J Public Health Manag Pract. 21(Suppl 2), S102-06. PubMed https://doi.org/10.1097/PHH.0000000000000170

36. Council of State and Territorial Epidemiologists (CSTE). Driving Public Health in the Fast Lane: The Urgent Need for a 21st Century Data Superhighway. 2019 [cited 2020 April 9]; Available from: https://resources.cste.org/data-superhighway/mobile/index.html.

37. McNabb SJ, et al. 2017. Informatics enables public health surveillance. J Health Spec. 5, 5559. https://doi.org/10.4103/jhs.JHS 28 17

38. Keller, R. COVID-19 shows why we must prioritize public health surveillance funding. 2020 [cited 2020 April 12].

39. American Medical Informatics Association (AMIA). COVID-19 Legislation Funding, Programs, and Provisions of Interest. 2020 [cited 2020 April 12]; Available from: https://www.amia.org/sites/default/files/COVID-19-Legislation-Chart.pdf.

40. U.S. Department of Health and Human Services. Welcome to HealthData.gov. [cited 2020 April 2]; Available from: https://healthdata.gov/.

41. Schomberg JP, et al. 2016. Supplementing Public Health Inspection via Social Media. PLoS One. 11(3), e0152117. PubMed https://doi.org/10.1371/journal.pone.0152117

42. Effland T, et al. 2018. Discovering foodborne illness in online restaurant reviews. J Am Med Inform Assoc. 25(12) PubMed https://doi.org/10.1093/jamia/ocx093 
43. Harris JK, et al. 2017. Using Twitter to Identify and Respond to Food Poisoning: The Food Safety STL Project. J Public Health Manag Pract. 23(6), 577-80. PubMed https://doi.org/10.1097/PHH.0000000000000516

44. Harris JK, et al. 2018. Evaluating the Implementation of a Twitter-Based Foodborne Illness Reporting Tool in the City of St. Louis Department of Health. Int J Environ Res Public Health. 15(5). PubMed https://doi.org/10.3390/ijerph15050833

45. Tucker CA, Larkin SN, Akers TA. 2011. Food Safety Informatics: A Public Health Imperative. Online J Public Health Inform. 3(2). PubMed https://doi.org/10.5210/ojphi.v3i2.3832 